\title{
Word association norms: Stability of response and chains of association'
}

\section{SYDELLE STONE SHAPIRO}

AUSTEN RIGGS CENTER

Word association norms are reported for 200 boys and girls, aged $12 \mathrm{yr} .9$ mos., to $13 \mathrm{yr} .8$ mos. For those words which constituted a retest, analysis showed a high consistency in the primary response. When the primaries were used as $S$ words, subjects frequently responded with the word that had originally produced the primary. In 14 cases, however, a new association was produced and these "chains of association" are presented.

The present study reports the results of a word association (WA) test given to eighth grade boys and girls. There were 82 words used in this study. Of these, 35 represent a retest (Shapiro, 1963), and 47 are either primaries (the most frequent response), secondaries (the second most frequent response), or tertiaries (the third most frequent response), selected from the 1963 norms and used in this study as stimulus words. Of interest is a comparison of results obtained in the retest with those of the test given to eighth graders two years previously. Of further interest is a content analysis of responses to those words which were primaries in the earlier study.

Method

A written single association WA test was given to 270 eighth grade boys and girls. It was conducted in the school cafeteria and all Ss took it as a group. Three different orders of words were used and an equal number of booklets in each order was distributed. The words were arranged in two columns, 30 to a page, with each word followed by a line on which the response was to be written. Instructions were read to the group and testing began immediately after. Ss were told to write the first word they thought of when they saw the stimulus word. Ss were urged to work rapidly and, to encourage speed, were instructed to write the time of completion of the test on the back of the booklet.

Of the 270 Ss tested, 200 protocols were selected. Ss were eliminated who were at the extremes of the age range and then Ss were randomly eliminated until 100 boys and 100 girls between the ages of $12 \mathrm{yr} .9$ mos. and 13 yr. 8 mos. remained.

Results

The associations for boys and girls were analyzed separately and are available from the author. In addition to a content and frequency analysis of primaries, secondaries, and tertiaries, the number of idiosyncratics and the total number of lexicographic and gramatically different responses are given.

Test-retest comparison. Test-retest comparisons of the responses to the 35 words common to the earlier test and the present study yielded the following: (a) Identical primaries were recorded in 28 instances for males and 29 instances for females. In every case but one (i.e. "stab" as the primary response to "jab" on the retest for females), those primaries which did change had become the secondaries or tertiaries. (b) The absolute frequency of the primary increased on the retest. Mean frequency of the primaries on the test was 40.31 for males and 39.43 for females; the retest frequencies were 45.23 and 47.83 . This increase was found to be significant for females $(t=2.2 ; \mathrm{df}=160$; $p<.05)$ but not for males $(t=1.3 ; d f=160)$. (c) The relative frequency of the primaries remained substantially the same on the retest. The Pearson coefficient of correlation for test-retest on frequency of the primaries was .71 for males and .62 for females.

Associations to primaries. WA were obtained for 32 words which had been found to beprimaries of stimulus words in the earlier test. Of interest was the use of the resultant norms to develop chains of association. The

Table 1. Chains of Primary Associations: Words in II are Primaries of I, Words in III are Primaries of II

\begin{tabular}{|c|c|c|c|c|c|}
\hline & 1 & \multicolumn{2}{|c|}{ II } & \multicolumn{2}{|c|}{111} \\
\hline DIM & $\begin{array}{l}\quad .37^{a} \rightarrow \\
-.10^{-}\end{array}$ & LIGHT & $.40 \rightarrow$ & DARK & $(.15)^{b}$ \\
\hline FUR & $\begin{array}{r}.51 \rightarrow \\
-.13\end{array}$ & COAT & $25 \rightarrow$ & HAT & $(.00)$ \\
\hline GAY & $\begin{array}{l}.52 \rightarrow \\
\leftarrow .30\end{array}$ & HAPPY & $.46 \rightarrow$ & SAD & $(.19)$ \\
\hline $\mathrm{H} \mid \mathrm{T}$ & $\begin{array}{l}.20 \rightarrow \\
\leftarrow .07\end{array}$ & BALL & $.20 \rightarrow$ & BAT & $(.05)$ \\
\hline HUT & $\begin{array}{r}.46 \rightarrow \\
-.06\end{array}$ & HOUSE & $.26 \rightarrow$ & HOME & $(.02)$ \\
\hline$J A B$ & $\begin{array}{r}.19 \rightarrow \\
-.03\end{array}$ & KNIFE & $26 \rightarrow$ & CUT & $(.01)$ \\
\hline LUG & $\begin{array}{r}.28 \\
\leftarrow .01\end{array}$ & CARRY & $.20 \rightarrow$ & HOLD & $(.01)$ \\
\hline MAY & $\begin{array}{l}.20 \\
\leftarrow .09\end{array}$ & MONTH & $.33 \rightarrow$ & YEAR & $(.00)$ \\
\hline MOW & $\begin{array}{r}.33 \rightarrow \\
-.21 \\
\end{array}$ & LAWN & $.38 \rightarrow$ & GRASS & $(.10)$ \\
\hline NOT & $\begin{array}{r}.21 \rightarrow \\
\leftarrow .04\end{array}$ & $\mathrm{NO}$ & $.80 \rightarrow$ & YES & $(.07)$ \\
\hline PAY & $\begin{array}{r}.53 \rightarrow \\
\leftarrow .12 \\
\end{array}$ & MONEY & $.16 \rightarrow$ & DOLLA & $(.00)$ \\
\hline RAW & $\begin{array}{r}.39 \rightarrow \\
\leftarrow .08 \\
\end{array}$ & MEAT & $.17 \rightarrow$ & STEAK & $(.01)$ \\
\hline RIB & $\begin{array}{r}30 \rightarrow \\
\leftarrow .10 \\
\end{array}$ & BONE & $.44 \rightarrow$ & DOG & $(.00)$ \\
\hline WAG & $\leftarrow .57 \rightarrow$ & TAIL & $.34 \rightarrow$ & $\operatorname{DOG}(\mathrm{S})$ & $(.14)$ \\
\hline
\end{tabular}

a. See text for explanation of figures

b. $p$ of words in III being given as responses to words in.I 
associations to 18 of the 32 primaries were reciprocals, i.e., the orginal stimulus word. For example the primary to "bad" is "good." When "good" is the stimulus word its primary is "bad." Associations to 14 of the words resulted in new words as primaries. Table 1 shows these chains as prepared from the current WA norms. The numbers indicate strength of association as a probability $(p)$ measure. Thus the pof the primary "light" occurring as a response to "dim" is .37; the $\mathrm{p}$ of the primary "dark" occurring as a response to "light" is .40 . The $\mathrm{p}$ of the reciprocal response to "light," i.e. "light-dim" is .10. Finally the p of "dark" being given as a response to "dim" is .15 . Discussion

The stability of normative responses so far as content of the primary is concerned would appear to be upheld. There is some evidence however of increase in the frequency of the primary. Jenkins \& Russell (1960) noted this increase in comparing WA norms collected at the University of Minnesota in 1927 and 1952. The increase observed in the present study occurred over a two year period. This increase may be the result of differences in test procedures. The present study emphasized speed and required single associa- tions. The earlier study was a multiple association test. The Ss had 17 seconds to respond to each word with up to five responses (Shapiro, 1963).

Speed instructions have been shown to increase the tendency to respond with the most popular response (Horton, Marlowe, \& Crown, 1963) and may account for the effect noted in this replication.

\section{References}

Horton, D. L., Marlowe, D., \& Crowne, D. P. The effect of instructional set and need for social approval on commonality of word association responses. J. abnorm. soc. Psychol., 1963. $66,67-72$.

Jenkins, J. J., \& Russell, W. A. Systematic changes in word association norms: 1910-1952. J. abnorm. soc. Psychol., 1960, 60, 293-304.

Shapiro, S. S. Word associations to CVCs by grade-school-aged children. Amherst: Univ. of Massachusetts, Dept. of Psychology, 1963.

\section{Note}

1. This research was supported by grant HD 01014-01 from the National Institute of Child Health and Human Development. Appreciation is expressed to Mr. James P. Reynolds, superintendent of schools and Mr. William A. Tully, principal of South Junior High School, Pittsfield, Mass. Author's address: Austen Riggs Center, Stockbridge, Mass.

\section{Comment on The Role of the Intertrial Interval in Serial Learning: A Clarification by Louis G. Lippman and M. Ray Denny}

The existence of a primacy effect in continuous serial learning (no ITI) has been substantiated, using a considerable variety of materials and procedures (Eysenck, 1959; Glanzer \& Peters, 1962; Breckenridge, Hakes, \& Young, 1965; Harcum \& Coppage, 1965; Lippman \& Denny, 1964). Lippman and Denny have shown that primacy effects occur when $S$ can view an item as first on trial 1 only (our group $E_{1}$ ) but do not occur when the first item is disguised by the presence of four extralist items which precede the "first" item only on the very first trial (our group $E_{2}$ ). In both instances the serial position curves were plotted from the first item of the list, i.e., the first item seen in group $E_{1}$ and the fifth seen in group $E_{2}$ on trial 1. In this journal, Keppel (1965) appears to regard the results of these two procedures as equivalent: "the continuous-list condition ... did not produce primacy and recency effects when position curves were plotted from the item appearing first on trial 1..." (p. 471). We would like to set the record straight on this count, at least with respect to primacy effects.

Harcum and Coppage (this journal) appear to share a similar interpretation of our results. They assert that because acquisition was terminated after 24 trials (prior to Ss' reaching a perfect trial criterion in groups $\mathrm{E}_{1}$ and $E_{2}$ ) the primacy effects had insufficient opportunity to develop. Our point here is that primacy effects, conventionally plotted, did show up in the $\mathrm{E}_{1}$ group even though they were not present in $E_{2}$. And the fact that groups $E_{1}$ and $E_{2}$ learned at approximately the same rate is not necessarily contradictory. Lack of facilitation from primacy in the $E_{1}$ group is in keeping with the finding that $\mathrm{Ss}$ of group $\mathrm{E}_{2}$ generated their own idiosyncratic primacy effects ("objective first" curves in our study). In other words, primacy can occur within 24 trials and there is nothing inconsistent in the fact that the two groups $\left(E_{1}\right.$ and $\left.E_{2}\right)$ learned at the same rate (primacy was really operative for both). Admittedly, there may have been insufficient trials for recency effects to accrue.

Although the presence or absence of dummy items preceding the first trial seems to represent a slight procedural difference, the difference in S's perception of the list (i.e. "first" item or selection of an anchor) appears considerable. In continuous serial learning, S actually sees the first item as first; therefore, an anchor is available for S's use (analogous to an objective standard in end-anchoring). When dummy items precede the first trial, however, the anchor is not clearly present. To use an anchor, S must note the reappearance of a particular syllable or find a syllable which is idiosyncratically meaningful. He can then use this item as his anchor (analogous to a subjective standard in judgment). Thus, we contend, the only appreciable difference between groups in which dummy items are present or absent is the consensus with which Ss select a particular item for an anchor. Such a contention is supported by the "objective first" curves found by Breckenridge et al and by us. These curves suggest that all Ss learn essentially the same way but employ different anchors. 